\title{
Rural Cancer Care: The Role of Space and Place in Care Delivery
}

\author{
Andrew P. Loehrer, MD, MPH ${ }^{1,2}$, Carrie H. Colla, PhD $^{2}$, and Sandra L. Wong, MD, MS ${ }^{1,2}$ \\ ${ }^{1}$ Department of Surgery, Geisel School of Medicine at Dartmouth, Dartmouth-Hitchcock Medical Center, Lebanon, NH ; \\ ${ }^{2}$ The Dartmouth Institute for Health Policy and Clinical Practice, Geisel School of Medicine at Dartmouth, Lebanon, NH
}

The gap in life expectancy between rural and urban Americans has increased 500\% since 1970, in part due to disparate access to and receipt of cancer care. ${ }^{1}$ In their publication, Obeng-Gyasi and colleagues describe the association between rural residence and patient presentation with, treatment for, and outcomes from care for women with breast cancer between 2004 and $2015 .^{2}$ The study finds that rurality was associated with increased cancer stage at the time of diagnosis, decreased the odds of undergoing breast reconstruction (odds ratio [OR] 1.25, 95\% confidence interval [CI] 1.19-1.30) and reduced overall survival (hazard ratio [HR] 0.92, 95\%CI 0.89-0.95).

The authors build on an extensive body of literature evaluating disparities in cancer care from race and ethnicity to insurance-based and broader socioeconomic inequities. Nonwhite patients have a significantly higher probability of being diagnosed with late-stage breast cancer, are less likely to receive optimal care, and have worse overall survival even controlling for stage at diagnosis. ${ }^{3-5}$ Similarly, uninsured and Medicaid beneficiaries more frequently present with later-stage disease and have worse outcomes compared with better insured patients. While knowledge of race- and insurance-based disparities has grown in recent years, our understanding of how space and place influence care delivery remain strikingly lacking.

The current study uses the National Cancer Database (NCDB), which captures approximately $70 \%$ of all new cancer diagnoses in the United States, to evaluate breast

(C) Society of Surgical Oncology 2020

First Received: 6 March 2020;

Published Online: 19 March 2020

S. L. Wong, MD, MS

e-mail: Sandra.L.Wong@dartmouth.edu cancer-the most commonly diagnosed malignancy and second leading cause of cancer mortality in women. There were differences across the continuum of care, suggesting rurality is contributing to inequitable processes and outcomes at multiple points of care delivery. In addition to later stage at diagnosis, increasing rurality was associated with decreased probability of reconstruction after mastectomy and worse stage-specific mortality. Similar rates of mastectomy and higher rates of radiation in rural areas after breast conservation together suggest that some of the process measures associated with Commission on Cancer accreditation are being met, yet timely diagnosis and overall survival are still lagging.

However, the results must be considered in the context of limitations that inform opportunities for future work to build on this study. First, the $70 \%$ of patients captured by NCDB is limited to patients who receive care at the larger (and often less rural) Commission on Cancer (CoC) accredited programs, as acknowledged by the authors. It is unknown whether these results are generalizable to the thousands of breast cancer patients who are diagnosed at and receive care at non-CoC hospitals. Second, the study population includes greater than $74 \%$ non-Hispanic white patients, differing significantly from the general population (60\% non-Hispanic white). ${ }^{6}$ Even after accounting for slightly lower rates of breast cancer in Hispanic women, the study sample is not representative of the U.S. breast cancer population. ${ }^{7}$ Such biases should be considered when contextualizing the full extent of intersectionality of disparities by race, place, and space. Third, additional spatial factors, beyond the rural vs urban place in which patients reside, are needed to better capture the heterogeneity of both urban and rural communities. These spatial measures include local area employment, poverty, segregation, and income inequality, to name a few. Targeting policy and system-level improvement will require complete populations, spatial data beyond geographic rural versus urban 
place, and data on sites of care delivery for precision, all in addition to population-specific measures to target programs and efforts.

Disparities in cancer care are multifactorial and include a combination of upstream social determinants as well as health system factors that guide access to, receipt of, and outcomes from care. Rural cancer disparities have been inadequately studied, especially with regard to incorporating the intersectionality with other factors, including income, insurance, race/ethnicity, and additional geospatial measures of inequality and broader socioeconomic deprivation. In order to drive population improvement in cancer outcomes, both hospital-level and comprehensive population level data are needed. This may require utilization of both claims and registry data that capture different, although complimentary aspects of care delivery for all patients, regardless of site of care. Furthermore, we need to evaluate the full heterogeneity of rurality. Rural Mississippi is not the same as rural Massachusetts, and small metropolitan areas may differ significantly in transportation options from isolated rural settings. In order to dissect this heterogeneity, however, we need datasets that capture all payers, ages, and sites of care delivery while simultaneously being able to incorporate additional geospatial measures to better capture the diversity of rural communities and their transportation and community building challenges. With health systems and policymakers alike focusing on rural disparities, collectively we can move the needle toward more equitable cancer care for all Americans.

DISCLOSURE The authors have no disclosures no conflicts of interest.

\section{REFERENCES}

1. Singh GK, Siahpush M. Widening rural-urban disparities in life expectancy, U.S., 1969-2009. Am J Prev Med. 2014;46(2):e19e29.

2. Obeng-Gyasi S, Timsina L, Bhattacharyya O, Fisher CS, Haggstrom DA. Breast cancer presentation, surgical management and mortality across the rural-urban continuum in the National Cancer Database. Ann Surg Oncol. 2020. https://doi.org/10.1245/s10434020-08376-y.

3. Ko NY, Hong S, Winn RA, Calip GS. Association of insurance status and racial disparities with the detection of early-stage breast cancer. JAMA Oncol. 2020;6(3):385-392. https://doi.org/10.1001/ jamaoncol.2019.5672.

4. Singh GK, Williams SD, Siahpush M, Mulhollen A. Socioeconomic, rural-urban, and racial inequalities in US cancer mortality: Part I-all cancers and lung cancer and part II-colorectal, prostate, breast, and cervical cancers. $J$ Cancer Epidemiol. 2011;2011:107497. https://doi.org/10.1155/2011/107497.

5. Nurgalieva ZZ, Franzini L, Morgan RO, Vernon SW, Liu CC, Du XL. Impact of timing of adjuvant chemotherapy initiation and completion after surgery on racial disparities in survival among women with breast cancer. Med Oncol. 2013;30(1):419. https://d oi.org/10.1007/s12032-012-0419-1.

6. U.S. Census Bureau (2016). U.S. Census Bureau QuickFacts: United States. https://www.census.gov/quickfacts/fact/table/US/P ST045218. Accessed 3 Mar 2020.

7. U.S. Cancer Statistics Working Group. U.S. Cancer Statistics Data Visualizations Tool, based on November 2018 submission data (1999-2016): U.S. Department of Health and Human Services, Centers for Disease Control and Prevention and National Cancer Institute; www.cdc.gov/cancer/dataviz, June 2019.

Publisher's Note Springer Nature remains neutral with regard to jurisdictional claims in published maps and institutional affiliations. 